OPEN ACCESS

Citation: D. Bennato (2020) mondo governato da dati e algoritmi. La data literacy attraverso il cinema. Media Education 11(1): 5-13. doi: 10.36253/ me-9090

Received: April 2020

Accepted: May 2020

Published: July 2020

Copyright: (c) 2020 D. Bennato. This is an open access, peer-reviewed article published by Firenze University Press (http://www.fupress.com/me) and distributed under the terms of the Creative Commons Attribution License, which permits unrestricted use, distribution, and reproduction in any medium, provided the original author and source are credited.

Data Availability Statement: All relevant data are within the paper and its Supporting Information files.

Competing Interests: The Author(s) declare(s) no conflict of interest.

\section{Un mondo governato da dati e algoritmi. La data literacy attraverso il cinema}

\author{
A world gorverned by data and algorithms. \\ Data literacy through cinema
}

\author{
Davide Bennato \\ Dipartimento di Scienze Umanistiche, Università di Catania \\ E-mail: davide.bennato@unict.it
}

\begin{abstract}
La società digitale pone al proprio centro l'utilizzo delle tecnologie che incorporano ogni processo individuale e collettivo. Nuove infrastrutture della società contemporanea sono gli algoritmi che definiscono le dinamiche sociali producendo dati, vere materie prime di questa condizione. Tutto ciò rende necessario una competenza relativamente nuova, la data literacy, intesa come capacità di leggere e comprendere i dati. Per formare in questa competenza il cinema può essere uno strumento utile, in particolare i film che hanno messo i dati al centro della narrazione.
\end{abstract}

Parole chiave: cinema, data literacy, dati, società digitale.
Abstract. The digital society focuses on the use of technologies that incorporate every individual and collective process. New infrastructures of contemporary society are the algorithms that define social dynamics by producing data, true raw materials of this condition. All this necessitates a relatively new competence in data literacy, understood as the ability to read and understand data. To train in this competence, cinema can be a useful tool, in particular the films that put data at the center of the story.

Keywords: cinema, data literacy, data, digital society.

\section{LA SOCIETÀ DIGITALE E LE SUE FONDAMENTA: SOGGETTI SOCIALI, PROCESSI SOCIALI, TECNOLOGIE SOCIALI}

Uno dei campi più frequentati dell'immaginario dal cinema contemporaneo è senza dubbio relativo ai film che mettono al centro della narrazione il rapporto con le tecnologie. Tradizionalmente questi film sono appannaggio della fantascienza proprio perché è nella fantascienza che meglio si costruisce il rapporto ambiguo con le tecnologie, ma negli ultimi tempi sono sempre più presenti forme di narrazione che usano altri generi - thriller, spy story, commedia - per dipanare il complesso rapporto che abbiamo con i disposi- 
tivi tecnici. E la cosa sta prendendo piede anche in Italia, tradizionalmente resistente alla narrazione centrata sulla tecnologia. Basti pensare che uno dei film di maggiore successo delle passate stagioni cinematografiche è stato Perfetti sconosciuti (Genovese, 2016) che racconta le vicissitudini di un gruppo di amici che durante una cena a mo' di sfida decidono di rivelare pubblicamente il contenuto di messaggi e telefonate che riceveranno durante la serata, trasformando così l'incontro in una sorta di processo di disvelamento della propria identità, rivelando così che il telefonino in realtà è uno strumento che nasconde alcuni dei segreti più inconfessabili che ci appartengono. Questo è solo uno dei casi che è possibile citare su come la cinematografia degli ultimi anni abbia cominciato a rappresentare la nostra dimensione sociale pesantemente incorporata nella componente tecnologica, mostrando come la tecnologia sia diventata un tema costante della nostra quotidianità che con la nostra quotidianità costringe a fare $i$ conti.

Alla luce di quanto detto, i film che mettono al loro centro la tecnologia sono film interessanti in quanto, avendo abbandonato l'impostazione speculativa tipica della narrazione fantastica, hanno cominciato ad usare l'iperrealismo come strumento per accedere ad una descrizione del nostro rapporto con le tecnologie e le relative conseguenze individuali e sociali.

Lo scopo di questo saggio è l'analisi di alcune delle pellicole cinematografiche che hanno posto al centro della loro rappresentazione la narrazione dei dati per mostrare come attraverso il cinema sia possibile educare ad un pensiero critico nei confronti della nostra società basata su dati e algoritmi. L'ipotesi di lavoro è che i dati possono essere considerati come delle nuove componenti mediali della società contemporanea, pertanto la narrazione che li riguarda può essere utile per una educazione critica alla consapevolezza che può essere considerata come parte di una più ampia data literacy.

Cominciamo con il definire contesto e terminologie che sono utili per comprendere per quale motivo i dati possano essere visti come una nuova forma di infrastruttura della società contemporanea. Per prima cosa dobbiamo presentare il concetto di società digitale.

La letteratura sociologica più recente, ha cominciato a introdurre diverse denominazioni che cercano di focalizzare l'attenzione sulla centralità che la tecnologia ha assunto negli ultimi anni: network society, connective society, platfom society, società digitale (Bennato, 2018b; Castells, 2002; van Dijk, 2002; van Dijck et al., 2019; Granieri, 2006; Rainie \& Wellman, 2012). Ogni definizione ha delle sue specificità e concetti chiave che declinano in maniera piuttosto dettagliata le caratteristiche socio-tecniche della struttura sociale contemporanea.
Ciononostante è possibile fare una sintesi generale di queste posizioni, in modo da sottolineare quali sono le fondamenta costitutive di quella che per semplicità chiameremo società digitale. La regola base è che la società digitale nasce dall'intersezione fra lo spazio/tempo fisico e lo spazio/tempo digitale.

In primo luogo il soggetto sociale che agisce. Da questo punto di vista possiamo dire che le diverse impostazioni sono d'accordo nel ritenere che il soggetto che agisce nella società digitale sia dotato di una doppia articolazione. Da un lato sia un soggetto che vive in uno spazio fisico, in continuità con le proprietà degli individui descritti dalla riflessione sociale del XIX e del XX secolo, da un altro è un soggetto che vive all'interno degli spazi immateriali definiti dalle diverse tecnologie digitali che costellano la società digitale. È un soggetto sociale che può essere individuale - come l'attore sociale - o collettivo - come i gruppi o le istituzioni, ma entrambi vivono nella doppia dimensione fisica e digitale.

In secondo luogo i processi sociali. Con questo termine facciamo riferimento ai diversi modi con cui $i$ soggetti sociali agiscono nella società digitale. Esistono diverse dinamiche, ma essenzialmente sono due i vincoli all'interno del quale i processi prendono forma. Da un lato le dinamiche di rete, ovvero il fatto che ogni soggetto fa parte di un intricato reticolo di altri soggetti macro o micro sociali - che ne limitano o potenziano l'agire a seconda del grado di potere di cui il soggetto gode. Dall'altro lato le dinamiche di flusso, ovvero quei processi che sono frutto delle dinamiche informazionali tipiche del digitale e che hanno conseguenze sia sullo spazio fisico che sullo spazio digitale. Un esempio in questo senso è la dimensione dell'accesso: una categoria che può essere compresa solo se la si considera contemporaneamente proprietà dello spazio fisico e dello spazio digitale.

In terzo luogo le tecnologie sociali. Per agire nella società secondo i processi che la caratterizzano, è necessario considerare la presenza delle tecnologie digitali che grazie alla loro proprietà di abilitare comportamenti sociali individuali e collettivi possono essere rubricate come tecnologie sociali (Marres, 2017, pp. 45-77). Le tecnologie sociali si presentano sottoforma di due strutture tecniche che ne definiscono caratteristiche e applicazioni. Una è la forma artefatto, con una propria fisicità e delle specifiche caratteristiche tecnologiche (per esempio: telefonini, consolle di videogiochi, smart t $v$ e così via dicendo). L'altra forma è quella immateriale di prodotti e servizi digitali (social media, servizi di streaming, app eccetera) che possiamo definire piattaforme. Entrambe però essendo incorporate nelle tecnologie digitali sono governate da algoritmi, 
ovvero da regole computazionali che vincolano sia il funzionamento che le conseguenze sociali del loro uso, ed entrambe producono dati, ovvero il flusso di informazioni che consente il funzionamento degli algoritmi e l'interoperabilità con altre tecnologie (artefatti o piattaforme). Quindi le tecnologie digitali possono essere fisiche - artefatti - o informazionali - piattaforme - che funzionano grazie agli algoritmi e proprio grazie ad essi producono dati.

Qui arriviamo ad un punto centrale del nostro ragionamento: l'importanza di dati e algoritmi. In estrema sintesi, se le tecnologie sociali rendono possibili tanto i processi sociali quanto l'attività dei soggetti sociali, allora è legittimo considerarle infrastrutture della società digitale. In pratica la società digitale è resa possibile dai gruppi e dalle persone che la abitano (soggetti), dalle dinamiche individuali o collettive che avvengono in essa (processi), ma soprattutto dalle tecnologie che rendono tutto questo possibile. Questa posizione non è particolarmente innovativa: che le infrastrutture abbiano un ruolo chiave all'aumentare del grado di tecnologizzazione della società è qualcosa che è possibile rilevare già con i social media (Bennato, 2011) così come ha senso considerare le tecnologie della società digitale come delle vere e proprie istituzioni sociali (Bennato, 2013).

Questa osservazione è molto importante per almeno due ordini di motivi.

In primo luogo la questione degli algoritmi come vincoli. Lazione sociale nella misura in cui prende forma tanto nello spazio fisico quanto nello spazio digitale, è vincolata ad entrambi gli spazi, ovvero deve rispettare sia le regole dello spazio fisico che le regole dello spazio digitale. Nello spazio fisico, le regole sono dettate dalla fisica, nello spazio sociale, le regole sono dettate dagli algoritmi che comunque sono frutto della sintesi fra norme tecniche e scelte del programmatore: in pratica sono parzialmente dei costrutti sociali incorporati in oggetti tecnici.

La seconda questione - centrale - è quella dei dati come onnipervasivi. Se gli algoritmi sono alla base delle tecnologie siano esse artefatti o piattaforme, vuol dire che i dati sono ovunque. In questo senso i dati sono sia prodotti tecnici, sia prodotti sociali così come le tecnologie sono tanto prodotti tecnici quanto prodotti sociali. In pratica i dati altro non sono che media necessari all'attuazione dei processi delle tecnologie digitali ma allo stesso tempo parte fondante della contemporanea ontologia sociale. Detto molto schematicamente: i dati fanno parte dall'attuale organizzazione sociale così come i concetti di identità, azione, istituzione e collettività.

La conseguenza di tutto ciò è chiara: se educare alla tecnologia è educare agli artefatti o alle piattaforme, educare ai dati vuol dire educare alla capacità di leggere il mondo circostante con la consapevolezza del ruolo giocato dagli algoritmi. La media education ci ha aperto la strada alla media literacy insistendo sul fatto che i media non sono riflesso della realtà ma loro interpretazione (Buckingham, 2006; Masterman, 1997), adesso è arrivato il momento della data literacy, perché i dati non sono neutrali, ma sono frutto di tecnologie prodotte specificamente all'interno di un processo di costruzione sociale.

\section{LA DATA LITERACY: LEGGERE I DATI PER INTERPRETARE IL MONDO}

Uno dei più classici adagi del mondo dell'apprendimento, recita che la scuola serve ad imparare a leggere, scrivere e far di conto. Se volessimo attualizzare queste competenze, sicuramente "far di conto" potrebbe essere declinata come numeracy, ma la competenza rispetto alla conoscenza numerica in senso contemporaneo si definisce più correttamente data literacy.

Vediamo questo punto in dettaglio.

Numeracy è il termine internazionale che serve per rendere l'abilità matematica ed è andato istituzionalizzandosi nel 1959 con il rapporto Crowther nel Regno unito a cui in tempi più recenti hanno fatto seguito $i$ diversi framework dell'OECD come i test PISA (Performance International Student's Assessment, 2017) per quanto riguarda la scuola secondaria o le prove PIAAC (Programme for the International Assessment of Adult Competencies, 2012) per quanto riguarda la formazione degli adulti (Raffaghelli, 2018). Per i test PISA la numeracy è intesa come la capacità di risolvere problemi matematici associando la capacità di ragionare espressa in termini sia matematici che quantitativi, mentre nel caso delle prove PIAAC viene descritta come la capacità di usare e comunicare concetti matematici da applicare in contesti di problem solving legati alla vita quotidiana. Nel complesso, le abilità matematiche di base sono essenzialmente connesse prevalentemente con le competenze aritmetiche mentre altri aspetti - misurazione, statistica - rimangono fondamentalmente esclusi (Raffaghelli, 2018). Esistono tentativi di superare questa visione ristretta della competenza sui dati introducendo il concetto statistical literacy (alfabetizzazione statistica) che dovrebbe prendere forma nella capacità di manipolare concetti come raccolta dei dati e campionamento, analisi dei dati associata a idee come inferenza e correlazione, capacità di comprendere informazioni numeriche organizzate attraverso la rappresentazione grafica dei dati (Gould, 2017). Una competenza così caratteriz- 
zata, per quanto accurata, comunque risente della tradizione novecentesca della statistica tradizionale, dato che non prende in considerazione l'esistenza di nuove forme approccio ai dati come nel caso dei big data, oppure nuove problematiche come quelle legate alla privacy del dato. Per questo motivo si suggerisce l'idea di avvicinarsi alla cultura del dato seguendo la cultura degli algoritmi (Breiman, 2001; Striphas, 2015) per fare in modo che queste competenze siano sempre più vicine alle necessità espresse da una società che manipola dati alla velocità delle tecnologie digitali (Gould, 2017).

Questo dibattito crea il contesto ottimale per introdurre il concetto di data literacy.

Con questo termine si indica la capacità che rende gli individui in grado di accedere, interpretare, valutare criticamente, gestire, maneggiare e usare eticamente i dati (Calzada-Prado \& Marzal, 2013; Koltay, 2015; Maybee \& Zilinski, 2015). Come si può notare da questa definizione iniziale, questo concetto è molto più vicino alla cultura del dato in un contesto da società digitale. L'accesso è un'idea connessa alla possibilità di poter entrare in possesso dei dati, la lotta alle cui disuguaglianze è compito del movimento open data. L'interpretazione sottolinea che nonostante siano dati e quindi frutto di rilevazioni e misurazioni, è necessario un processo di comprensione che pur usando tecniche statistiche e quantitative siano comunque frutto dell'esperienza dell'utilizzatore. La valutazione critica del dato è un concetto che se da un lato si definisce come qualità del dato, dall'altro implica la consapevolezza delle forme con cui il dato è stato prodotto, socialmente e tecnologicamente. Gestire e maneggiare i dati sono due tematiche che sono interne alla dimensione logistica dell'informazione strutturata che rimandano al fatto che i dati si trovano stoccati in database o comunque archivi più $o$ meno digitali. Infine la modernità di questa competenza è espressa dall'uso etico dei dati. Etica dei dati vuol dire essenzialmente che i dati non sono solo costrutti inerti che fungono da materia prima di altri processi (conoscitivi, decisionali, valutativi), ma riguardano sia $\mathrm{i}$ soggetti sociali a cui fanno riferimento (individui o istituzioni poco importa) sia le informazioni che è possibile desumere da essi e che potrebbero portare un esercizio eccessivo di potere da parte di chi li usa (Bennato, 2014). Quello che rende interessante la data literacy, è che i concetti di cui è formata sono perfettamente compatibili con l'attuale riflessione critica sui big data e sulle conseguenze etiche del loro utilizzo (Bennato, 2015).

Esistono diversi modi per declinare il concetto di data literacy, tanto più che la sua istituzionalizzazione è sicuramente molto recente. Per trovare degli elementi comuni nelle diverse indicazioni che vengono date su come realizzare percorsi di data literacy al di là della definizione generale - che comunque risulta essere piuttosto condivisa - può essere utile usare un approccio empirico che prende le mosse dall'analisi dei framework presenti nella letteratura di settore. Gli elementi che si trovano sono: la consapevolezza (comprendere cosa sono i dati e qual è il loro ruolo nelle comunità o nelle società), l'accesso (comprendere come identificare, localizzare e usare in modo appropriato i database e i dataset intesi come organizzazione strutturate di dati), il coinvolgimento (valutare, analizzare, organizzare e interpretare i dati esistenti e prendere decisioni basate sui dati), la gestione (pianificare e gestire i dati, compreso lo sviluppo di protocolli per la sicurezza, stoccaggio, condivisione, documentazione), la comunicazione (sintetizzare, visualizzare e rappresentare i dati), l'uso etico (riconoscere le fonti dei dati, valutare e gestire i rischi, comprendere le tematiche coinvolte nel riutilizzo dei dati), preservazione (essere consapevoli delle pratiche di curatela per l'archiviazione a lunga scadenza) (Maybee \& Zilinski, 2015).

Un'altra metodologia - anch'essa empirica - per provare a isolare le diverse tipologie di approccio verso la data literacy è quella che prende le mosse sempre dalla letteratura sull'argomento, ma preferisce avvalersi di una strategia computazionale a partire dai termini chiave estratti in maniera digitale. In questo caso gli approcci principali alla data literacy sono quattro e si caratterizzano per avere un proprio specifico focus: il focus sulla ricerca (si basa sulle dimensioni relative alla ricerca e considera la data literacy come un aspetto della più ampia information literacy e si rivolge essenzialmente ai professionisti che si confrontano con tali concetti come i bibliotecari, lasciando spazio a concetti come l'uso dei metadati e il riuso dei dati), il focus sull'aula (qui il centro è la formazione degli studenti e il percorso per renderli competenti nel trattamento dei dati nell'apprendimento problem-based), il focus sulla dimensione "artigianale"l (intendendo in questo caso sull'uso di strumenti concreti per migliorare le capacità di analisi dei dati), il focus sull'inclusione (dove i dati sono usati per superare le diseguaglianze nella conoscenza, per abilitare i membri della comunità ad accedere ai dati per i proprie preoccupazioni) (Matthews, 2016).

Come si è potuto vedere, la data literacy porta con sé diverse impostazioni dal punto di vista del framework e diversi approcci secondo quanto previsto dalla letteratura, ma possiamo sostenere tranquillamente che tutte queste sfaccettature intercettano lo stesso campo con-

\footnotetext{
${ }^{1}$ Con questo concetto rendiamo il termine "carpentry" usato originariamente nel paper, letteralmente carpenteria/falegnameria, connotato in senso metaforico.
} 
cettuale all'interno del quale è possibile elaborare una strategia formativa di consapevolezza sull'importanza e sull'utilizzo dei dati. Da questo punto di vista risulta essere particolarmente utile la definizione di data literacy condivisa in letteratura che non solo la considera una competenza pratica (accedere, gestire, maneggiare) ma anche una attitudine mentale (interpretare, valutare criticamente, usare eticamente).

Per quanto riguarda gli orizzonti formativi faremo nostre le parole di Juliana Elisa Raffaghelli: «Tentando una sintesi, i traguardi formativi di tipo più generico in relazione ai data literacy sono:

- Saper collegare problemi dell'ambito disciplinare nel quale si lavora o si apprende con il bisogno di evidenza empirica basata su dati esistenti, da estrarre o da generare.

- Saper distinguere la diversa natura dei dati.

- Saper navigare attraverso informazioni basate su dati, interpretando correttamente grafici e tabelle.

- Saper riconoscere situazioni in cui i dati vengono utilizzati in modo inadeguato, e in particolar modo quanto possono essere stati manipolati per supportare opinioni o concetti tendenziosi.

- Riconoscere la differenza della comunicazione basata su dati in relazione ai target comunicativi a cui si rivolge.

- Conoscere strumenti di base per trattare i dati.

- Saper comunicare in modo efficace attraverso l'uso di dati e in relazione al target a cui si rivolge la comunicazione.

Invece, le competenze più specifiche sono:

- Saper distinguere la diversa natura dei dati, riconoscendo le architetture di dataset e database in base ai problemi esplorati.

- Conoscere e saper utilizzare strumenti e approcci di modellizzazione statistica per l'analisi dei dati.

- Conoscere e saper utilizzare strumenti e approcci per la costruzione avanzata e interattiva (e.g., webapps) di forme di visualizzazione dei dati elaborati.

- Conoscere e saper utilizzare le opportunità offerte dai big e open data come base per la generazione di servizi e prodotti» (Raffaghelli, 2018, pp. 106-107).

\section{IL CINEMA DEI DATI: NON SOLO DIGITALE}

Ci sono diversi motivi per considerare il cinema un ottimo strumento per avviare al tema della data litera$c y$. In primo luogo la lunga tradizione che vuole il cinema come un medium privilegiato nei processi educativi, così come evidenziato dal paradigma delle arti popolari della media education (Masterman, 1997), e nonostante l'approccio contemporaneo abbia ridimensionato la prevalenza dello sguardo cinematografico a favore di un approccio più complesso e meno discriminatorio (Rivoltella, 2001), per alcuni aspetti il cinema - ma più in generale l'audiovisivo - ha una sua capacità di penetrazione nei processi sociali di comprensione e apprendimento (Manovich, 2002; Malavasi, 2005). In secondo luogo perché la narrazione cinematografica contemporanea si confronta con una serie di temi che pur rispettando il canone del cinema come fenomeno culturale ed artistico, riesce a dare una chiave di lettura interessante della forma culturale contemporanea e rivelare alcune dinamiche soprattutto legate al nostro rapporto con la tecnologia (per esempio: Bennato, 2018a). Infine perché i dati sono molto difficili da rappresentare in quanto non hanno né forma né dimensione, sono visualizzabili solo grazie alle strategie di raccolta e di rappresentazione grafica, pertanto l'unico modo per accedere ad una riflessione che renda chiaro il loro ruolo e le conseguenze sulla vita quotidiana è senza dubbio una strategia di rappresentazione visiva, di cui il cinema è una di queste, sicuramente la più importante.

A questo punto cominceremo a introdurre una serie di film che nel loro sviluppo narrativo hanno dipanato alcuni temi che consideriamo chiave per un percorso di data literacy che faccia proprie tutte le suggestioni della condizione socio-tecnica della società digitale.

Dal punto di vista metodologico, la decisione di introdurre i film che seguiranno è stata dettata da tre considerazioni. Per prima cosa abbiamo scelto questi film perché sono una buona panoramica di come la cinematografia contemporanea abbia affrontato il tema dell'impatto sociale dei dati. Siamo consapevoli che la scelta qui presentata sia tacciabile di arbitrarietà: possiamo dire che questo piccolo gruppo rappresenta un insieme di film più ampio che è attualmente in corso di raccolta e catalogazione. Poi abbiamo preferito dare prevalenza a film molto famosi o comunque prodotti cinematografici destinati al grande pubblico. Esistono alcune pellicole che hanno affrontato il tema dei dati in maniera interessante, ma sono film che adottano un linguaggio eccessivamente di nicchia da poter essere considerati utili in un percorso di data literacy, per esempio $\pi$. Il teorema del delirio (Aronofsky, 1998). Infine abbiamo scelto di lasciare spazio a pellicole in cui il trattamento della trama è distante da rappresentazioni fantastiche ma sia più vicino alle forme dell'iperrealismo. Il motivo che ci ha guidati in questa scelta è essenzialmente didattico: riteniamo che i dati siano un tema già di per sé complesso che una narrazione fantascientifica rischierebbe di distrarre dalla loro cogente attualità. 
Il primo film di questo nostro excursus è sicuramente The bank (Connolly, 2001). Il film racconta la storia del brillante matematico Jim Doyle (David Wenham) che sta lavorando su un software basato sulla matematica dei frattali con il quale cerca di prevedere l'andamento della borsa. Questo progetto viene intercettato da Simon O’Reilly (Anthony La Paglia), amministratore delegato di una banca di investimenti piuttosto potente che fornisce a Doyle mezzi e strumenti praticamente illimitati per usare il suo sofwtare nelle speculazioni di borsa. Il tutto si intreccia con i problemi di un piccolo paese dell'Australia in cui a causa della crisi economica i residenti stanno perdendo le proprie case, pignorate dalle banche. Dal punto di vista della data literacy, questo film è interessante per diversi motivi. Molto affascinante la rappresentazione dei dati: le immagini in cui si vedono i frattali sono matematicamente piuttosto plausibili oltre a dare concretezza ad un concetto spesso difficile da rappresentare con quello dell'analisi tecnica nel mondo della finanza. Interessante è anche l'ambientazione della vicenda nel mondo delle banche: questo ambito è sicuramente quello che meglio rappresenta l'attuale fame di dati delle organizzazioni data-based, ma evidenzia come l'uso indiscriminato delle informazioni finanziarie possano avere conseguenze nel mondo reale. Inoltre non bisogna dimenticare che oggi i film su banche, finanza e informazioni finanziarie sono diventati un genere a sé stante grazie anche allo shock culturale dovuto alla crisi dei subprime dei 2006 a cui fece seguito la recessione economica del 2007, ma all'epoca dell'uscita del film questo tema sembrava essere semplicemente una speculazione, frutto di un'idea originale degli autori del soggetto Brian Price e Mike Betar. Tutto ciò è ancora più interessante se si considera che una sottotrama del film affronta il tema del pignoramento dei piccoli proprietari di case insolventi, ovvero la stessa situazione che ha innescato la crisi economica del 2007.

Un altro film interessante e con una ambientazione decisamente inusuale per parlare di dati e delle loro conseguenze sociali è S1m0ne (Niccol, 2002). La storia prende le mosse dalle vicissitudini di un regista caduto in disgrazia Viktor Taransky (Al Pacino) il quale dopo una serie di film senza successo e alla ricerca di ispirazione, viene contattato da Hank Aleno (Elias Koteas), un suo fan che gli consegna un pacco piuttosto misterioso prima di morire. Dentro questo pacco c'è un hard disk che contiene un sofisticato software che consente a Taranski di creare un'attrice virtuale (Rachel Roberts) che chiamerà Simone (crasi di simulation one, il nome del software) con il quale non solo sarà in grado di girare delle pellicole di enorme successo, ma diventerà la beniamina del pubblico, cosa che provocherà non pochi problemi quando deciderà di porre fine a questo strano esperimento. Questo film è l'unica concessione alla narrazione fantascientifica di questa lista, ma la sua ambientazione in una Hollywood piuttosto credibile lo rende un'occasione molto utile per discutere di alcune delle conseguenze sociali dei dati. In primo luogo il film dà del processo di costruzione del personaggio virtuale una rappresentazione piuttosto potente attraverso in una scena in cui Taranski dà vita a Simone mediante una complessa libreria di attori e attrici i cui volti e le cui espressioni sono accuratamente campionate e archiviate in un enorme database. Inoltre nel momento in cui Simone viene lanciata come attrice grazie alla magia del cinema e al mistero che circonda la sua figura, diventa la beniamina di tutti: giornalisti, mondo di Hollywood e pubblico, tanto che nessuno è più disposto a credere alla sua inesistenza quando Taranski cercherà di rivelare l'inganno. Questo sviluppo della trama è molto interessante poiché porta a riflettere sul tema della realtà delle simulazioni: è possibile considerare reale una cosa che esiste solo e unicamente nel mondo digitale? Tema questo non solo tipico della riflessione sulla società dello spettacolo e sulla realtà delle simulazioni (Baudrillard, 1980; Debord, 2004), ma anche di un certo tipo di cinematografia di quegli anni, che aveva riscontrato un grosso successo di pubblico e critica con il film The Truman show (Weir, 1998), che con S1m0ne condivide non solo i temi ma anche il regista (Niccol è l'autore del soggetto del film di Weir). La struttura narrativa del film riprende l'archetipo del Pigmalione e lo attualizza ambientandolo nel mondo cinematografico (come È nata una stella di William Wellman, pellicola del 1937), ma la riflessione sulla realtà del virtuale è di particolare modernità, considerando per esempio il recente dibattito sui deepfake, ovvero video in cui i volti e i movimenti delle persone sono interamente simulati al computer tramite una intelligenza artificiale creando video profondamente realistici (Bennato, 2019).

Un ambito che sembra lontano dall'impatto sociale dei dati è sicuramente il mondo dello sport, con le sue tematiche legate alla fatica, al sacrificio, alla voglia di vincere. Ma è proprio quest'ultimo elemento che - visto dalla prospettiva del management - legittima i dati come strumento strategico. È questa in estrema sintesi il plot del film Moneyball (Miller, 2011) che si ispira alle vicende della squadra di baseball Oakland Athletics. La storia racconta del general manager Billy Beane (Brad Pitt) che cerca di risollevare la propria squadra di baseball da un periodo caratterizzato da una serie interminabile di sconfitte, e per farlo si rivolge alla dirigenza a cui chiede un aumento di budget che gli viene negato. Mentre è alla ricerca di una soluzione, incontra Peter Brand (Jonah 
Hill), un giovane laureato in economia a Yale che gli propone un approccio diverso per costruire una squadra di giocatori buoni ma non eccezionali sfruttando fino all'ultimo dollaro di budget a sua disposizione. L'idea è quella di analizzare statisticamente le performance di ogni giocatore e di opzionarne l'acquisto prima che le squadre si accorgano del loro reale valore. Nonostante la palese ostilità di tutti, Beane e Brand mostreranno di avere ragione e di ottenere risultati mai raggiunti dall'Oakland. Fra tutti i film di questa lista, Moneyball è senza dubbio quello che meglio rappresenta l'impatto sociale dei big data. Per prima cosa il riferimento alla sabermetrics: è l'analisi quantitativa del baseball attraverso la statistica che deve il suo nome alla società che raccoglie questi dati (SABR: Society for American Baseball Research). Questo elemento introduce nella trama la questione della decisione basata su dati, che è molto difficile da rappresentare ma affascinante da raccontare in un progetto di data literacy. In più il film mostra come i dati possano avere un impatto sociale rappresentato da un mondo come quello dello sport che sembrerebbe lontano dalla data analytics e che invece se ne può avvantaggiare se opportunamente utilizzato. Infine il film è tratto da una storia vera e raccontata nell'omonimo libro di Michael Lewis, cosa che lo rende una interessante testimonianza di come il mondo dei dati possa cambiare il nostro punto di vista sulle cose.

Dopo il 2011, i film in cui una parte della trama è basata su dati diventano più presenti nelle sale cinematografiche, complice la diffusione delle idee legate ai big data ma soprattutto alle conseguenze economiche nefaste della conoscenza matematica applicata in maniera spregiudicata al mondo della finanza. Infatti non è un caso che nel 2015 escono due film assolutamente straordinari per un trattamento sofisticato del tema e per un percorso di data literacy. Il primo è The big short (McKay, 2015). Il film racconta in maniera romanzata - così come il libro da cui è tratto - le vicissitudini di un gruppo di persone variamente legate al mondo della finanza e degli investimenti, che riuscirono ad ottenere profitti multimilionari perché furono in grado di prevedere il crollo dei mutui subprime e la relativa crisi economica degli anni successivi. Il film deve la sua bellezza non solo perché è una produzione da blockbuster hollywoodiano per via del cast stellare - Christian Bale, Steve Carell, Ryan Gosling, Brad Pitt - ma ha degli elementi di cultura dei dati che lo rendono molto affascinante. In primo luogo la trama racconta le vicende da un punto di vista umano, ma in diversi momenti ha degli approfondimenti legati al linguaggio della finanza in cui i tecnicismi vengono spiegati facendo delle piccole incursioni che usano il linguaggio della divulga- zione in un contesto narrativo molto pop. Per esempio, in una scena davvero iconica, l'attrice Margot Robbie interpreta se stessa in una vasca da bagno in cui spiega le caratteristiche dei mutui subprime, oppure in un'altra sequenza il premio Nobel Richard Taler e l'attrice Selena Gomez ad un tavolo di un casinò di Las Vegas spiegano i CDO sintetici. In secondo luogo il film è tratto da una inchiesta giornalistica piuttosto dettagliata di Michael Lewis, lo stesso autore del libro Moneyball, che indagando sul tema introduce un punto di vista molto interessante come il bias di conferma o l'ideologia del "too big to fail" 2 che portò la finanza statunitense a non vedere l'arrivo della crisi economica del 2007. In sintesi il film racconta la speculazione delle banche alla base della crisi economica, non solo mostrando l'uso spregiudicato di strumenti finanziari ma spiegando anche le conseguenze dal punto di vista della valutazione tecnica, ovviamente nei limiti di un film hollywoodiano.

Se The big short racconta la crisi economica alle origini della contrazione economica la cui onda lunga abbiamo vissuto anche recentemente, un altro film uscito lo stesso anno invece cambia registro e contesto ma fa capire la capacità che hanno i dati di interpretare il mondo in cui viviamo senza scomodare big data o tecnologie digitali connesse. Stiamo parlando di Spotlight (McCarty, 2015), film che prende le mosse dall'indagine del gruppo di giornalisti del Boston Globe che li condusse alla scoperta di un complesso giro di preti pedofili nella città di Chicago e coperto dalla locale Arcidiocesi cattolica, che valse al giornale il premio Pulitzer nel 2003. Il film usa i toni del thriller giornalistico con ampie concessioni alla narrazione investigativa tipica dei film di indagine per evidenziare il complesso sistema di silenzi e taciti accordi che gravitavano intorno alla vicenda e che ne hanno impedito che venisse scoperta anni prima nonostante ci fossero stati tutti i segnali. Il film si presta ottimamente per un percorso di data literacy per diverse ragioni, come la presenza di attori straordinari - tra gli altri Michael Keaton e Mark Ruffalo e una sceneggiatura che assieme al titolo di miglior film valse al progetto la premiazione agli Oscar del 2016. Per esempio l'essenzialità del lavoro basato su dati: se è vero che oggi il tema del giornalismo basato su dati è molto più diffuso grazie anche alla presenza di strumenti digitali (Antenore \& Splendore, 2017), il film mostra come un buon reportage possa essere fatto anche con semplice carta e penna, valorizzando la dimensione metodologica rispetto alla dimensione tecnologica. Il film inoltre

\footnotetext{
${ }^{2}$ Letteralmente "troppo grande per fallire": è un termine giornalistico entrato nel linguaggio comune per descrivere la sottostima dei rischi da parte di banche come Lehman Brothers che nonostante la loro dimensione, sono state profondamente colpite dalla crisi dei mutui subprime.
} 
mostra come una analisi basata su dati possa dare un quadro molto dettagliato di una situazione. La scena rivelatrice in questo senso è quando il gruppo di giornalisti di Spotlight - il team investigativo del Boston Globe - in una conferenza telefonica con Richard Sipe, lo psicologo consulente del caso, scoprono che alla luce dei dati relativi ai pattern di comportamento, i preti pedofili coinvolti sarebbero dovuti essere molti di più, il $6 \%$ dei sacerdoti dell'Arcidiocesi di Boston, ovvero 90 preti invece dei 13 che il team aveva già scoperto ${ }^{3}$.

Proviamo a fare il punto del tipo di formazione ai dati di questo percorso cinematografico.

In primo luogo la dimensione professionale dell'uso dei dati. I film mostrano come in alcuni contesti professionali il ricorso ai dati faccia parte integrante della cultura di quel settore - la finanza, il giornalismo - mentre in altri ambiti il ricorso ai dati è molto più recente ma molto più produttivo come nel cinema o nello sport.

In secondo luogo la dimensione decisionale nell'uso dei dati. Tutti i film mostrano come un uso opportuno dei dati permetta di fare analisi di scenario (The big short, Spotlight), analisi previsionali (The bank), valutazioni operative (Moneyball), sviluppo di prodotti (S1m0ne).

In terzo luogo la dimensione relativa all'impatto sociale. Ognuno dei film in modi diversi mostrano come i dati possano avere delle conseguenze profonde nella vita delle persone, sia a livello circoscritto (le vicissitudini di una squadra di baseball, la vita sociale di una città americana) sia a livello globale (l'industria cinematografica, l'economia globale).

Infine la componente etica. I dati sono un potere tecnico incorporato nelle organizzazioni (finanza, giornalismo) o nelle professioni (general manager, regista cinematografico) il cui uso può avere delle ripercussioni profonde sulle persone destinatarie di tali processi e in quanto tale richiedono una enorme responsabilità nell'uso e nel trattamento.

In conclusione possiamo dire che la data literacy è una competenza piuttosto giovane entrata nel linguaggio della media education e che ha dovuto trovare una propria definizione che la rendesse autonoma da concetti simili - ma più restrittivi - come numeracy o statistical literacy. Se leggere, scrivere e far di conto definivano la cittadinanza nel XIX secolo, adesso il far di conto si è evoluto nella competenza del confrontarsi con i dati che, non solo sono dappertutto come informazioni veicolate, basti pensare al sovraccarico di numeri, cifre e statistiche che attraverso la stampa stiamo assistendo in questo periodo di quarantena da coronavirus, ma sono dapper-

\footnotetext{
${ }^{3}$ Il dato è uno dei risultati della ricerca condotta dallo psicologo sul comportamento sessuale dei sacerdoti (Sipe, 1990).
}

tutto come materia prima degli algoritmi che stanno alla base dei processi della società digitale. Per questo motivo è necessario introdurre un percorso di data literacy ad ogni livello formativo perché la consapevolezza dei dati è una consapevolezza di libertà e di comprensione di processi che potrebbero restare opachi. In questo senso il cinema può essere un ottimo strumento per rappresentare le forme culturali di questo mondo e rendere coscienti della complessità della società contemporanea.

\section{BIBLIOGRAFIA}

Antenore, M., \& Splendore, S. (Eds). (2017). Data Journalism. Guida essenziale alle notizie fatte con $i$ numeri. Mondadori.

Aronofsky, D. (Director) (1998). $\pi$. Il teorema del delirio [Translated title] [Film]. Arrisan Entertainement.

Baudrillard, J. (1980). Simulacri e impostura. Cappelli.

Bennato, D. (2011). Sociologia dei media digitali. Relazioni sociali e processi comunicativi del web partecipativo. Laterza.

Bennato, D. (2013). Istituzioni tecnologiche e partecipative. I social media come istituzioni sociali. In M. Cacioppo \& S. Severino (Eds.), La prossimità a distanza. Contributi psicosociali per lo studio degli usi, abusi e dipendenze nel Web 2.0 (pp. 70-86). FrancoAngeli.

Bennato, D. (2014). Etica dei Big data. Le conseguenze sociali della raccolta massiva di informazioni. Studi culturali, 11(1), 86-92.

Bennato, D. (2015). Il computer come macroscopio. Big data e approccio computazionale per comprendere $i$ cambiamenti sociali e culturali. FrancoAngeli.

Bennato, D. (2018a). Non è un episodio, non è marketing: è la realtà. Black Mirror come non adattamento alla tecnologia. In D. Bennato (Ed.), Black Mirror. Distopia e antropologia digitale (pp. 19-64). Villaggio Maori Edizioni.

Bennato D. (2018b). La sociologia digitale. in D. Chieffi (Eds.), Comunicare digitale (pp. 26-41). Centro di documentazione giornalistica.

Bennato, D. (2019, 26 Settembre). Deepfake anche in Italia: mostro di una società senza più certezze. Agendadigitale.eu. https://www.agendadigitale.eu/culturadigitale/deepfake-anche-in-italia-il-mostro-di-unasocieta-senza-piu-certezze/

Breiman L. (2001). Statistical modeling: the two cultures, Statistical Science. 16(3), 199-231. http://dx.doi. org/10.1214/ss/1009213726

Buckingham, D. (2006). Media education. Alfabetizzazione, apprendimento e cultura contemporanea. Erickson. (Original work published 2003) 
Calzada Prado, J., \& Marzal, M. Á. (2013). Incorporating Data Literacy into Information Literacy Programs: Core Competencies and Contents. Libri, 63(2), 123134. http://dx.doi.org/10.1515/libri-2013-0010

Castells, M. (2002). La nascita della società in rete. EGEA. (Original work published 1996)

Connolly, R. (Director) (2001). The bank. [Film]. Arena Film Ply Ltd.

Debord, G. (2004). La società dello spettacolo. Baldini Castoldi Dalai. (Original work published 1967)

Genovese, P. (Director) (2016). Perfetti sconosciuti [Film]. Medusa Film.

Gould, R. (2017). Data Literacy is Statistical Literacy, Statistics Education Research Journal, 16(1), 22-25.

Granieri, G. (2006). La società digitale. Laterza.

Koltay, T. (2015). Data literacy: in search of a name and identity. Journal of Documentation, 71(2), 401-415. https://doi.org/10.1108/JD-02-2014-0026

Malavasi, P. (2005). Interpretare il testo filmico tra fascinazione e riflessione pedagogica. In P. Malavasi, S. Polenghi \& P. C. Rivoltella (Eds.), Cinema, pratiche formative, educazione (pp. 53-67). Vita e Pensiero.

Manovich, L. (2002). Il linguaggio dei nuovi media. Olivares. (Original work published 2001)

Marres, N. (2017). Digital sociology. The reinvention of social research. Polity press.

Masterman, L. (1997). A scuola di media, Brescia. La Scuola. (Original work published 1985)

Matthews, P. (2016). Data literacy conceptions, community capabilities. The Journal of Community Informatics, 12(3), 47-56.

Maybee, C., \& Zilinski, L. (2015). Data informed learning: A next phase data literacy framework for higher education, Proceedings of the Association for Information Science and Technology, 52(1), 1-4. https://doi. org/10.1002/pra2.2015.1450520100108

McCarty, T. (Director) (2015). Spotlight. [Film]. Open Road Films.

McKay, A. (Director) (2015). The big short [Film]. Plan B Entertainment.

Miller, B. (Director) (2011). Moneyball [Film]. Michael De Luca Productions.

Niccol, A. (Director) (2001). S1m0ne [Film]. New Line Cinema.

Raffaghelli, J. E. (2018). Oltre il "far di conto" nell'era digitale. La frontiera della data literacy. In M. Ranieri (Eds.). Teoria e pratica delle new media literacies (pp. 99-133). Aracne,

Rainie, L. \& Wellman, B. (2012). Networked. Il nuovo sistema operativo sociale. Guerini.

Rivoltella, P. C. (2001). Media education. Modelli, esperienze, profilo disciplinare. Carocci.
Sipe, R. (1990). A secret world: Sexuality and the search for celibacy. Routledge.

Striphas, T. (2015). Algorithmic culture, European Journal of Cultural Studies, 18(4-5), 395-412. https:/doi. org/10.1177/1367549415577392

Van Dijck, J., Poell, T., \& De Waal, M. (2019). Platform society. Valori pubblici e società connessa. Guerini. (Original work published 2018)

Van Dijk, J. (2002). Sociologia dei nuovi media. Il Mulino. (Original work published 1999)

Weir, P. (Director) (1998). The Truman show [Film]. Paramount Pictures. 